\title{
25. OCCURRENCE OF INOCERAMUS IN THE SOUTH ATLANTIC AND OXYGEN ISOTOPIC PALEOTEMPERATURES IN HOLE 530A ${ }^{1}$
}

\author{
E. J. Barron ${ }^{2}$ and E. Saltzman, Rosenstiel School of Marine and Atmospheric Research, Miami, Florida \\ and \\ D. A. Price, Department of Geology, University of Miami, Coral Gables, Florida
}

\begin{abstract}
Inoceramus occurs in every DSDP hole that penetrated Cretaceous sediments in the South Atlantic Ocean, and specimen occurrence has been mapped in detail for each core. Oxygen and carbon isotope measurements were completed on 18 Inoceramus specimens from Hole 530A. Textural evidence of diagenesis is accompanied by depletion in ${ }^{18} \mathrm{O}$. Paleotemperature results were obtained from 11 well-preserved specimens. Bottom water temperatures in the Angola Basin decreased from $23^{\circ} \mathrm{C}$ during the Coniacian to $13^{\circ} \mathrm{C}$ near the end of the Campanian.
\end{abstract}

\section{INTRODUCTION}

A major objective at Site 530 was to determine the paleoceanographic evolution of the Angola Basin, particularly during the Cretaceous episodes of black shale deposition. Determination of paleotemperatures is an integral part of reconstructing the paleoenvironmental history.

Most paleotemperature investigations have stressed isotopic analyses of foraminifers from deep-sea cores. Unfortunately, there is a notable lack of well-preserved foraminifers from Cretaceous sediments, despite the fact that many Deep Sea Drilling Project (DSDP) holes have penetrated Cretaceous sediments. As in sections elsewhere, there were no suitably preserved specimens of foraminifers found in the Cretaceous sediments in Hole 530A. However, there were abundant Inoceramus specimens, ranging in age from Maestrichtian to Coniacian, many of which were clearly well preserved.

The majority of quantitative paleotemperature estimates from the Cretaceous have been derived from oxygen isotopic analyses of belemnites and Inoceramus from outcrops of epicontinental sea deposits (e.g., Lowenstam and Epstein, 1954). The major advantage of analyses of these macrofossils is that the coarse crystalline structure is likely to preserve the isotopic signal. The major disadvantage is that the epicontinental-sea setting may be subject to more variable environmental conditions, such as fresh-water runoff, making the data difficult to interpret. Subaerial exposure may also result in substantial alteration. Because Inoceramus specimens preserved in deep sea cores do not have the same drawbacks as those in exposed continental sections, they have potential to expand greatly our knowledge of Cretaceous paleotemperatures.

We have mapped the distribution of Inoceramus fragments in all DSDP cores which penetrated Cretaceous

\footnotetext{
${ }^{1}$ Hay, w. W., Sibuet, J.-C., et al., Init. Repts. DSDP, 75: Washington (U.S. Govt. Printing Office).

2 Present address: National Center for Atmospheric Research, P.O. Box 3000, Boulder, CO 80307 .
}

sediments in the South Atlantic Ocean. Knowledge of the distribution of Inoceramus will aid in understanding their paleoecology as well as describe the potential source of isotopic data.

Oxygen and carbon isotope ratios were measured on Inoceramus fragments in various states of preservation in order to interpret diagenetic trends and establish textural criteria for selection of samples for paleotemperature analysis. This study resulted in paleotemperature data from 11 well-preserved Inoceramus fragments from Hole 530A, ranging in age from Coniacian to Campanian. These data confirm earlier studies of warm bottom water temperatures during the Cretaceous (see Savin, 1977 for view). Changes in bottom water sources during this time influenced the character of sedimentation in the Angola Basin. These changes were probably controlled by tectonic developments and eustatic sea level fluctuations in the early South Atlantic.

\section{OCCURRENCE OF INOCERAMUS IN SOUTH ATLANTIC CORES}

Inoceramus is an extinct epibenthic bivalve. The coarsely crystalline ostracum, which consists of vertically oriented calcite prisms, is preserved in deep-sea sediments (Fig. 1). Inoceramus is known to occur in a wide variety of paleoenvironments, within a large range of paleodepths and associated with a wide variety of sediment types (e.g., Thiede and Dinkelman, 1977). Studies of Inoceramus in the western interior seaway of North America (Kauffman, 1967) indicate that the organisms were adapted to soft mud bottoms.

Detailed mapping of the distribution of Inoceramus in DSDP cores will aid in understanding the paleoecology of this extinct organism. Rather than relying on descriptions of the occurrence of Inoceramus available in DSDP reports, which in some cases are rather general, all DSDP cores which penetrated Cretaceous sediments in the South Atlantic were examined specifically for Inoceramus. However, this does not insure a quantitative description of the organism's occurrence because visual examination is limited to the two faces of the split core. 


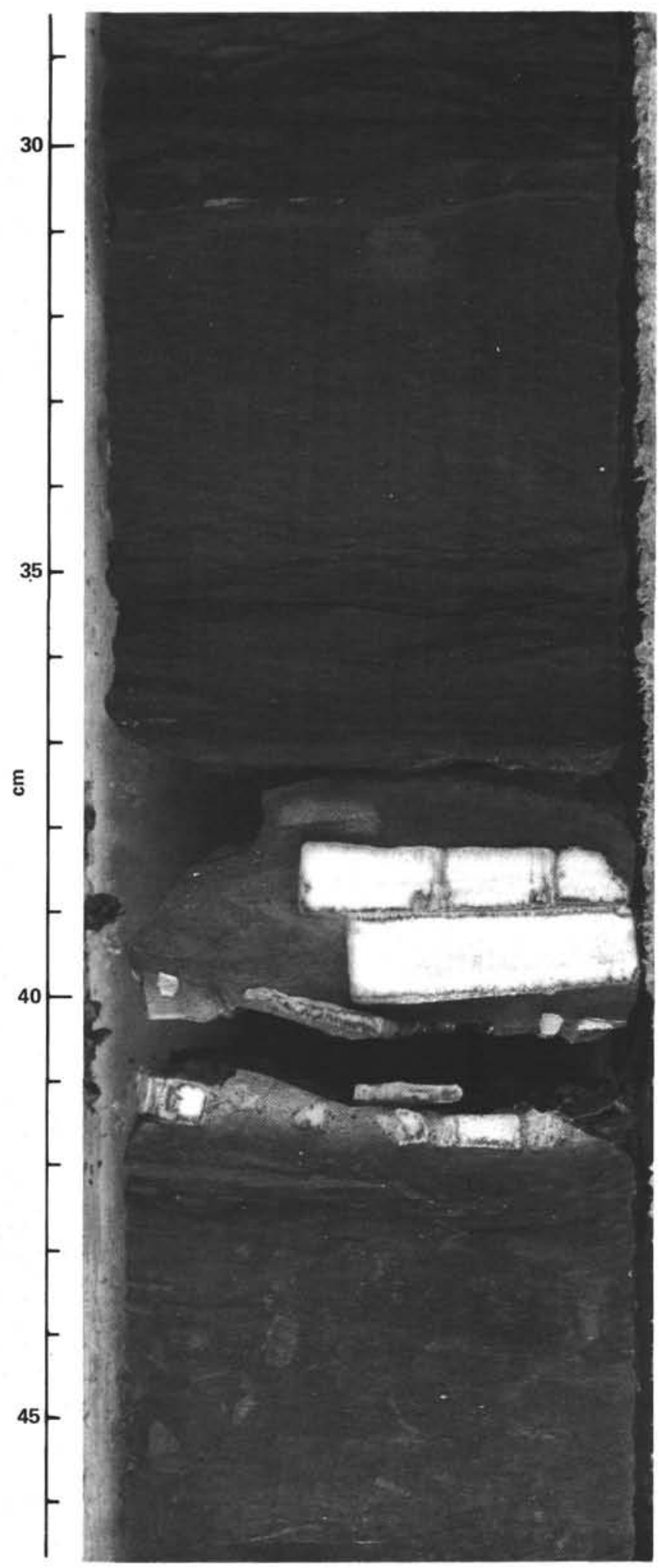

Figure 1. Example of preserved Inoceramus fragments at Samples $530 \mathrm{~A}-63-1,30-45 \mathrm{~cm}$.

A large amount of fossil material may be hidden (e.g., Kauffman, 1976). The specimens described in this chapter are typically fragments rather than whole shells, probably because of predation (E. Kauffman, pers. comm.). Individual prisms are not included as an occurrence.
Table 1 gives the occurrence of Inoceramus in South Atlantic DSDP cores, the number of fragments visible, the character of the preservation and a general designation of the sediment type. Samples collected for microscopic examination to determine their suitability for isotopic analysis are indicated by an asterisk. The stage designation is given from the respective site reports. Based on the stratigraphic zonation given in the site reports and the time scale of Obradovich and Cobban (1975) for the Late Cretaceous and van Hinte (1976a, b) for the Early Cretaceous and Jurassic, an estimated age in millions of years is calculated by assuming a constant sedimentation rate within paleontologic zones. This information is summarized in Table 2 . The occurrence of Inoceramus is plotted in Figure 2.

All of the holes that penetrated Cretaceous sediments have at least one Inoceramus occurrence. Although Inoceramus are widely distributed in the South Atlantic, the factors controlling distribution are unclear. For instance Sites 21, 356, and 357 are located in close proximity on the Rio Grande Rise and the Sao Paulo Plateau. Inoceramus is common in Campanian strata in cores from Sites 21 and 356, but is not common in cores from the geographically intermediate Site 357 . yet the geographically intermediate site, Site 357 , is not characterized by abundant Campanian Inoceramus while Sites 21 and 356 have common occurrences. The Rio Grande Rise was also the site of abundant Inoceramus during the Santonian-Coniacian. Both of the basinal sites ( 355 and $530 \mathrm{~A}$ ) appear to have abundant Inoceramus in the late Campanian but not in the early Campanian. It is possible that there is a hiatus at the Maestrichtian-Campanian boundary at Site 355, but this is of unknown duration. With the exception of Site 361 on the South African margin, which has poor stratigraphic control, all South Atlantic DSDP sites contain Inoceramus in Santonian sediments. Five sites penetrated the middle Cretaceous, of which three have Albian Inoceramus. Of course, all of these comparisons are dependent on the recognition of any hiatuses. The oldest occurrence of Inoceramus is in Oxfordian strata from from Site 330 on the Falkland Plateau.

Preservation varies widely, involving silicification, recrystallization, overgrowth, and, rarely, dissolution. Many specimens appear completely unaltered. Table 1 also indicates the variety of associated sediment types, including euxinic sediments, olive clays and nannofossil foraminiferal oozes.

Occurrences in the South Atlantic also represent a wide range of paleodepths. The presence of shallow water indicators at Site 21 (Maxwell et al., 1970) suggests a paleodepth of 100 to $400 \mathrm{~m}$ in the early Campanian, and the paleodepth of Inoceramus was probably not much deeper. In contrast, the paleodepth in Hole 530A during the Late Cretaceous is in the range of 3500 to $4500 \mathrm{~m}$, based on basement age and thermal subsidence history. Estimated paleodepths, derived from the respective DSDP reports, are given in Table 3.

It is important to note that the occurrence of Inoceramus in Hole 530A greatly extends the depth range as compared to Thiede and Dinkelman (1977). Careful examination of the distribution of turbidites (Stow, this 
Table 1. Inoceramus occurrences and samples for microscopic examination and isotopic analysis.

\begin{tabular}{|c|c|c|c|c|c|}
\hline $\begin{array}{l}\text { Core-Section } \\
\text { (interval in cm) }\end{array}$ & $\begin{array}{c}\text { No. of } \\
\text { occurrences }\end{array}$ & $\begin{array}{l}\text { Preservation } \\
\text { (visual exam.) }\end{array}$ & Sediment type & Stage & $\begin{array}{c}\text { Age } \\
\text { (m.y. ago) }\end{array}$ \\
\hline \multicolumn{6}{|l|}{ Leg 3, Hole 21} \\
\hline $4-4,130-131^{a}$ & 1 & Good & Nanno-foram ooze & 1. Campanian & 71.6 \\
\hline $4-5,105-107^{a}$ & $i$ & Good & Nanno-foram ooze & 1. Campanian & 72.1 \\
\hline $4-6,31-32^{\mathrm{a}}$ & i & Good & Nanno-foram ooze & 1. Campanian & 72.2 \\
\hline $5-1,21-22^{a}$ & 1 & Moderate & Nanno-foram ooze & 1. Campanian & 73.5 \\
\hline $5-2,69-70^{\mathrm{a}}$ & Several & Moderate & Nanno-foram ooze & 1. Campanian & 74.1 \\
\hline $5-2,73-75^{a}$ & 1 & Good & Nanno-foram ooze & 1. Campanian & 74.1 \\
\hline $5-2,76-82$ & 1 & Poor & Nanno-foram ooze & 1. Campanian & 74.1 \\
\hline $5-2,125-128^{\mathrm{a}}$ & 1 & Moderate & Nanno-foram ooze & 1. Campanian & 74.2 \\
\hline $5-3,53-58$ & $i$ & Poor & Nanno-foram ooze & 1. Campanian & 74.5 \\
\hline $5-3,93-99^{a}$ & 1 layer & Disaggregated & Nanno-foram ooze & 1. Campanian & 74.7 \\
\hline $5-5,20-21^{a}$ & 1 & Good & Nanno-foram ooze & 1. Campanian & 75.4 \\
\hline $5-5,39-41$ & 1 & Good & Nanno-foram ooze & 1. Campanian & 75.4 \\
\hline $5-5,69-71^{a}$ & 1 & Reduction, disaggregated & Nanno-foram ooze & 1. Campanian & 75.4 \\
\hline $5-5,69-71^{a}$ & i & Reduction, disaggregated & Nanno-foram ooze & 1. Campanian & 75.5 \\
\hline $5-5,83-84$ & 1 & Reduction, disaggregated & Nanno-foram ooze & 1. Campanian & 75.5 \\
\hline $5-5,90-91$ & $i$ & Reduction, disaggregated & Nanno-foram ooze & 1. Campanian & 75.5 \\
\hline $5-5,95-97^{a}$ & Several & Reduction, disaggregated & Nanno-foram ooze & 1. Campanian & 75.5 \\
\hline $5-5,121-122^{\mathrm{a}}$ & 1 & Good & Nanno-foram ooze & 1. Campanian & 75.7 \\
\hline $5-6,29-31^{\mathrm{a}}$ & 1 & Good to poor & Nanno-foram ooze & 1. Campanian & 75.8 \\
\hline $5-6,53-56^{a}$ & Several & Disaggregated & Nanno-foram ooze & 1. Campanian & 76.0 \\
\hline $5-6,64-70$ & 1 & Poor & Nanno-foram ooze & 1. Campanian & 76.0 \\
\hline $5-6,90-91$ & 1 & Poor & Nanno-foram ooze & 1. Campanian & 76.1 \\
\hline $5-6,94-95$ & 1 & Poor & Nanno-foram ooze & 1. Campanian & 76.3 \\
\hline $6-5,147-148^{\mathrm{a}}$ & i & Moderate & Nanno-foram ooze & e. Campanian & 78.0 \\
\hline $6-6,23-24$ & 1 & Poor & Nanno-foram ooze & e. Campanian & 78.1 \\
\hline $6-6,26-27$ & 1 & Poor & Nanno-foram ooze & e. Campanian & 78.1 \\
\hline $6-6,28-29$ & 1 & Poor & Olive nanno clay & e. Campanian & 78.1 \\
\hline $6-6,30-34^{a}$ & $i$ & Good & Olive nanno clay & e. Campanian & 78.1 \\
\hline $6-6,36-39^{a}$ & 1 layer & Good & Olive nanno clay & e. Campanian & 78.1 \\
\hline $6-6,45-47^{a}$ & 1 & Moderate & Nanno-foram ooze & e. Campanian & 78.1 \\
\hline $6-6,58-61^{\mathrm{a}}$ & 2 layers & Moderate & Nanno-foram ooze & e. Campanian & 78.1 \\
\hline $6-6,71-72^{a}$ & 1 & Good & Olive nanno clay & c. Campanian & 78.1 \\
\hline $6-6,81-82$ & i & Poor & Olive nanno clay & e. Campanian & 78.1 \\
\hline $6-6,106-107^{a}$ & $i$ & Moderate & Nanno-foram ooze & e. Campanian & 78.3 \\
\hline $6-6,106-114^{a}$ & Several & Moderate & Nanno-foram ooze & e. Campanian & 78.3 \\
\hline $6-6,114-116^{\mathrm{a}}$ & 1 & Moderate disaggregated & Nanno-foram ooze & e. Campanian & 78.3 \\
\hline $6-6,146-147$ & 1 & Poor & Nanno-foram ooze & e. Campanian & 78.4 \\
\hline $7-1,83-84$ & 1 & Reduction & Nanno-foram ooze & e. Campanian & 78.7 \\
\hline $7-2,97-99^{\mathrm{a}}$ & ? & Pelecypod? & Nanno-foram ooze & e. Campanian & 79.1 \\
\hline $7-2,101-102$ & Several & Poor & Nanno-foram ooze & e. Campanian & 79.1 \\
\hline $7-2,108-109$ & Several & Poor & Nanno-foram ooze & e. Campanian & 79.1 \\
\hline $7-2,123-125^{a}$ & 1 & Moderate & Nanno-foram ooze & e. Campanian & 79.1 \\
\hline $7-2,142-147^{a}$ & 1 & Good & Nanno-foram ooze & e. Campanian & 79.1 \\
\hline $7-3,45-46$ & 1 & Poor & Nanno-foram ooze & e. Campanian & 79.4 \\
\hline $7-4,84-85$ & 1 & Poor & Nanno-foram ooze & e. Campanian & 79.9 \\
\hline $7-6,75-76^{\mathrm{a}}$ & $i$ & Moderate & Nanno-foram ooze & e. Campanian & 80.4 \\
\hline $8-2,65-66^{a}$ & 1 & Good & Nanno-foram ooze & e. Campanian & 81.0 \\
\hline $8-3,120-122^{a}$ & i & Moderate & Nanno-foram ooze & e. Campanian & 81.6 \\
\hline \multicolumn{6}{|l|}{ Leg 36, Hole 327A } \\
\hline $12-3,64-74$ & ? & Poor & Olive gray clay & Maestrichtian & $\approx 67.0$ \\
\hline $14-4,134-135$ & i & Poor & Red brown clay & Cenomanian & 92.0 \\
\hline $14-4,136-137^{a}$ & 1 & Good & Red brown clay & Cenomanian & 92.0 \\
\hline $14-4,139-140$ & 1 & Poor & Red brown clay & Cenomanian & 92.0 \\
\hline $14-4,140-141$ & 1 & Poor & Red brown clay & Cenomanian & 92.0 \\
\hline $14-4,151-152^{a}$ & 1 & Good & Red brown clay & Cenomanian & 92.0 \\
\hline $14-5,3-4$ & 1 & Poor & Red brown clay & Cenomanian & 92.0 \\
\hline $14-5,4-5^{\mathrm{a}}$ & 1 & Moderate & Red brown clay & Cenomanian & 92.0 \\
\hline $14-5,5-6^{a}$ & $i$ & Poor to moderate & Red brown clay & Cenomanian & 92.0 \\
\hline $14-5,12-13^{a}$ & 1 & Poor to moderate & Red brown clay & Cenomanian & 92.0 \\
\hline $14-5,15-16$ & 1 & Poor & Red brown clay & Cenomanian & 92.0 \\
\hline $14-5,31-32^{\mathrm{a}}$ & 1 & Moderate & Red brown clay & Cenomanian & 92.0 \\
\hline $14-5,45-46$ & 1 & Poor & Red brown clay & Cenomanian & 92.0 \\
\hline $14-5,61-62^{\mathrm{a}}$ & i & Moderate & Red brown clay & Cenomanian & 92.0 \\
\hline $14-5,82-83^{a}$ & 1 & Moderate & Red brown clay & Cenomanian & 92.1 \\
\hline $14-5,86-87$ & 1 & Poor & Red brown clay & Cenomanian & 92.1 \\
\hline $14-5,94-95^{\mathrm{a}}$ & Several & Moderate & Red brown clay & Cenomanian & 92.1 \\
\hline $14-5,102-103^{\mathrm{a}}$ & 1 & Moderate & Red brown clay & Cenomanian & 92.1 \\
\hline $14-5,135-136^{\mathrm{a}}$ & 1 & Poor to moderate & Red brown clay & Cenomanian & 92.1 \\
\hline $14-5,142-143$ & 1 & Poor & Red brown clay & Cenomanian & 92.1 \\
\hline $15-2,130-137^{a}$ & 1 & Moderate & Light brown clay & 1. Albian & 95.8 \\
\hline $15-2,141-142$ & 1 & Poor & Light brown clay & I. Albian & 95.8 \\
\hline $15-2,145-146^{a}$ & 1 & Poor to moderate & Light brown clay & 1. Albian & 95.8 \\
\hline Leg 36, Hole 330 & & & & & \\
\hline $1-1,50-51^{a}$ & 1 & Moderate & Euxinic & e.-m. Albian & 100.0 \\
\hline $5-2,137-138^{a}$ & 2 layers & Poor & Euxinic & Oxfordian & 143.2 \\
\hline $6-1,129-130^{\mathrm{a}}$ & 1 & Moderate & Euxinic & Oxfordian & 143.9 \\
\hline $6-2,30-32^{a}$ & $i$ & Moderate & Euxinic & Oxfordian & 144.0 \\
\hline $6-2,34-35$ & $i$ & Moderate & Euxinic & Oxfordian & 144.0 \\
\hline $6-2,58-59^{a}$ & 1 & Moderate & Euxinic & Oxfordian & 144.0 \\
\hline $6-3,126-127$ & 1 & Poor & Euxinic & Oxfordian & 144.2 \\
\hline $6-5,104-105^{a}$ & i & Good & Euxinic & Oxfordian & 144.4 \\
\hline $7-2,20-21^{a}$ & 1 & Good & Euxinic & Oxfordian & 144.7 \\
\hline $7-3,10-11$ & $i$ & Poor & Euxinic & Oxfordian & 144.8 \\
\hline $7-3,14-15$ & 1 & Poor & Euxinic & Oxfordian & 144.8 \\
\hline $7-3,21-22$ & 1 & Poor & Euxinic & Oxfordian & 144.8 \\
\hline $7-3,47-48^{\mathrm{a}}$ & 1 & Good, disaggregated & Euxinic & Oxfordian & 144.9 \\
\hline $7-3,51-57^{a}$ & $i$ & Good & Euxinic & Oxfordian & 144.9 \\
\hline
\end{tabular}




\section{E. J. BARRON, E. SALTZMAN, D. A. PRICE}

Table 1. (Continued.)

\begin{tabular}{|c|c|c|c|c|c|}
\hline $\begin{array}{l}\text { Core-Section } \\
\text { (interval in cm) }\end{array}$ & $\begin{array}{c}\text { No. of } \\
\text { occurrences }\end{array}$ & $\begin{array}{l}\text { Preservation } \\
\text { (visual exam.) }\end{array}$ & Sediment type & Stage & $\begin{array}{c}\text { Age } \\
\text { (m.y. ago) }\end{array}$ \\
\hline \multicolumn{6}{|c|}{ Leg 36, Hole 330 Cont. } \\
\hline $\begin{array}{l}7-3,54-55^{\mathrm{a}} \\
7-3,55-56^{\mathrm{a}} \\
7-3,105-106 \\
7-3,132-133 \\
7-5,108-109^{\mathrm{a}} \\
7-6,18-19 \\
7-6,21-22^{\mathrm{a}} \\
7-6,43-45^{\mathrm{a}} \\
7-6,61-62^{\mathrm{a}} \\
7-6,75-76 \\
8-1,126-127 \\
8-3,27-28^{\mathrm{a}} \\
8-3,33-34 \\
8-3,45-46 \\
8-3,54-55 \\
8-3,88-89\end{array}$ & $\begin{array}{c}1 \\
1 \\
1 \\
1 \\
1 \\
1 \\
1 \\
1 \\
1 \\
1 \\
1 \\
1 \text { layer } \\
1 \\
1 \\
1 \\
1\end{array}$ & $\begin{array}{l}\text { Good } \\
\text { Moderate } \\
\text { Poor } \\
\text { Poor } \\
\text { Good, disaggregated } \\
\text { Good } \\
\text { Good } \\
\text { Good } \\
\text { Good } \\
\text { Poor } \\
\text { Poor } \\
\text { Good } \\
\text { Poor } \\
\text { Poor } \\
\text { Poor } \\
\text { Poor }\end{array}$ & $\begin{array}{l}\text { Euxinic } \\
\text { Euxinic } \\
\text { Euxinic } \\
\text { Euxinic } \\
\text { Euxinic } \\
\text { Euxinic } \\
\text { Euxinic } \\
\text { Euxinic } \\
\text { Euxinic } \\
\text { Euxinic } \\
\text { Euxinic } \\
\text { Euxinic } \\
\text { Euxinic } \\
\text { Euxinic } \\
\text { Euxinic } \\
\text { Euxinic }\end{array}$ & $\begin{array}{l}\text { Oxfordian } \\
\text { Oxfordian } \\
\text { Oxfordian } \\
\text { Oxfordian } \\
\text { Oxfordian } \\
\text { Oxfordian } \\
\text { Oxfordian } \\
\text { Oxfordian } \\
\text { Oxfordian } \\
\text { Oxfordian } \\
\text { Oxfordian } \\
\text { Oxfordian } \\
\text { Oxfordian } \\
\text { Oxfordian } \\
\text { Oxfordian } \\
\text { Oxfordian }\end{array}$ & $\begin{array}{l}144.9 \\
144.9 \\
144.9 \\
144.9 \\
145.2 \\
145.2 \\
145.2 \\
145.2 \\
145.3 \\
145.3 \\
147.2 \\
147.4 \\
147.4 \\
147.4 \\
147.4 \\
147.4\end{array}$ \\
\hline \multicolumn{6}{|l|}{ Leg 39 Site 355} \\
\hline $\begin{array}{l}17-2,137-138^{\mathrm{a}} \\
17-2,139-140^{\mathrm{a}} \\
17-3,8-9 \\
17-3,10-11 \\
17-4,95-96 \\
17-4,96^{\circ} \\
17-4,96-98 \\
17-4,104-105^{\mathrm{a}} \\
17-4,113-114^{\mathrm{a}} \\
17-4,120-121 \\
18-1,87-92^{\mathrm{a}} \\
18-1,104-105^{\mathrm{a}} \\
18-2,118-119^{\mathrm{a}} \\
18-3,17-19^{\mathrm{a}} \\
18-3,53-54^{\mathrm{a}} \\
18-3,61-62 \\
18-3,81-84^{\mathrm{a}} \\
19-2,75-83 \\
19-2,85-87^{\mathrm{a}} \\
19-2,100-101 \\
19-2,107-109^{\mathrm{a}} \\
19-2,116-117^{\mathrm{a}}\end{array}$ & $\begin{array}{c}1 \\
1 \\
1 \\
1 \\
1 \\
1 \text { layer } \\
\text { Several } \\
1 \\
1 \\
\text { Several } \\
1-7 \mathrm{~cm} \\
1 \\
1 \text { layer } \\
1 \\
1 \\
1 \\
1 \\
\text { Several } \\
\text { Several } \\
1 \\
1 \\
1\end{array}$ & $\begin{array}{l}\text { Good } \\
\text { Good } \\
\text { Poor } \\
\text { Poor } \\
\text { Poor } \\
\text { Good } \\
\text { Poor } \\
\text { Moderate } \\
\text { Moderate } \\
\text { Poor } \\
\text { Good } \\
\text { Good } \\
\text { Good } \\
\text { Moderate } \\
\text { Moderate } \\
\text { Poor } \\
\text { Moderate to good } \\
\text { Poor } \\
\text { Good } \\
\text { Poor } \\
\text { Marginal } \\
\text { Marginal }\end{array}$ & $\begin{array}{l}\text { Brown clay } \\
\text { Brown clay } \\
\text { Brown clay } \\
\text { Brown clay } \\
\text { Cemented clast } \\
\text { Brown clay } \\
\text { Brown clay } \\
\text { Brown clay } \\
\text { Brown clay } \\
\text { Brown clay } \\
\text { Brown clay } \\
\text { Brown clay } \\
\text { Brown clay } \\
\text { Brown clay } \\
\text { Brown clay } \\
\text { Brown clay } \\
\text { Brown clay } \\
\text { Brown clay } \\
\text { Brown clay } \\
\text { Brown clay } \\
\text { Brown clay } \\
\text { Brown clay }\end{array}$ & 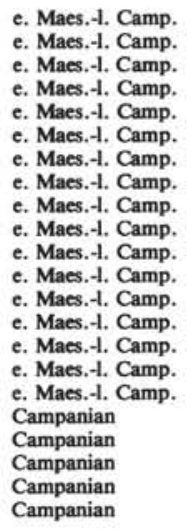 & $\begin{array}{l}69.7 \\
69.7 \\
69.7 \\
69.7 \\
70.1 \\
70.1 \\
70.1 \\
70.1 \\
70.1 \\
70.1 \\
70.6 \\
70.6 \\
70.9 \\
70.9 \\
71.1 \\
71.1 \\
71.2 \\
72.8 \\
72.8 \\
72.9 \\
72.9 \\
72.9\end{array}$ \\
\hline \multicolumn{6}{|l|}{ Leg 39, Hole 356} \\
\hline $\begin{array}{l}34-1,56-57 \\
34-1,57-58 \\
34-1,71-72 \\
34-1,73-74 \\
34-2,70-71 \\
34-2,124-126 \\
34-3,39-40^{\mathrm{a}} \\
34-3,42-43^{\mathrm{a}} \\
34-3,54-55^{\mathrm{a}} \\
34-4,31-33 \\
34-4,42-43 \\
34-4,67-68 \\
34-6,129-130 \\
34-6,135-136 \\
35-4,49-50 \\
36-4,11-12 \\
37-1,144-148 \\
38-2,9-12^{\mathrm{a}}\end{array}$ & $\begin{array}{c}1 \\
1 \\
1 \\
1 \\
1 \\
\text { Several } \\
1 \\
1 \\
1 \\
\text { Several } \\
1 \\
1 \\
1 \text { layer } \\
1 \\
1 \\
2 \\
\text { Several } \\
\text { Several } \\
\text { Several }\end{array}$ & $\begin{array}{l}\text { Silicified } \\
\text { Silicified } \\
\text { Silicified } \\
\text { Silicified } \\
\text { Silicified } \\
\text { Silicified } \\
\text { Poor to moderate } \\
\text { Poor to moderate } \\
\text { Poor to moderate } \\
\text { Silicified } \\
\text { Silicified } \\
\text { Silicified } \\
\text { Moderate } \\
\text { Poor } \\
\text { Poor } \\
\text { Poor } \\
\text { Poor } \\
\text { Poor } \\
\text { Poor }\end{array}$ & $\begin{array}{l}\text { Olive marl } \\
\text { Olive marl } \\
\text { Olive marl } \\
\text { Olive marl } \\
\text { Olive marl } \\
\text { Olive marl } \\
\text { Olive marl } \\
\text { Olive marl } \\
\text { Olive marl } \\
\text { Olive marl } \\
\text { Olive marl } \\
\text { Olive marl } \\
\text { Olive marl } \\
\text { Olive marl } \\
\text { Olive marl } \\
\text { Olive marl } \\
\text { Olive marl } \\
\text { Tan marl } \\
\end{array}$ & $\begin{array}{l}\text { Campanian } \\
\text { Campanian } \\
\text { Campanian } \\
\text { Campanian } \\
\text { Campanian } \\
\text { Campanian } \\
\text { Campanian } \\
\text { Campanian } \\
\text { Campanian } \\
\text { Campanian } \\
\text { Campanian } \\
\text { Campanian } \\
\text { Campanian } \\
\text { Campanian } \\
\text { 1. Santonian } \\
\text { 1. Santonian } \\
\text { 1. Santonian } \\
\text { e. Sant.- } \\
\text { 1. Coniac. } \\
\text { e. Sant-- }\end{array}$ & $\begin{array}{l}76.4 \\
76.4 \\
76.5 \\
76.5 \\
77.0 \\
77.1 \\
77.3 \\
77.3 \\
77.3 \\
77.7 \\
77.7 \\
77.8 \\
79.0 \\
79.0 \\
82.1 \\
83.0 \\
83.7 \\
84.1 \\
84.3\end{array}$ \\
\hline $\begin{array}{l}38-3,46-47 \\
38-3,71-73\end{array}$ & $\begin{array}{l}\text { Several } \\
\text { Several }\end{array}$ & $\begin{array}{l}\text { Poor } \\
\text { Poor }\end{array}$ & $\begin{array}{l}\text { Tan marl } \\
\text { Tan marl }\end{array}$ & $\begin{array}{l}\text { e. Sant.- } \\
\text { I. Coniac } \\
\text { e. Sant.- } \\
\text { l. Coniac }\end{array}$ & $\begin{array}{l}84.3 \\
84.3\end{array}$ \\
\hline $38-3,75-76^{a}$ & Several & Moderate to poor & Tan marl & $\begin{array}{l}\text { e. Sant.- } \\
\text { I. Coniac. }\end{array}$ & 84.3 \\
\hline $38-3,111-112^{a}$ & Several & Moderate to poor & Tan marl & $\begin{array}{l}\text { e. Sant.- } \\
\text { 1. Coniac. }\end{array}$ & 84.3 \\
\hline $38-3,132-133$ & 1 & Moderate to poor & Tan marl & $\begin{array}{l}\text { e. Sant.- } \\
\text { I. Coniac. }\end{array}$ & 84.3 \\
\hline $\begin{array}{l}39-2,78-79 \\
40-3,85-87\end{array}$ & 1 & $\begin{array}{l}\text { Clast } \\
\text { Clast }\end{array}$ & $\begin{array}{l}\text { Tan marl } \\
\text { Tan marl }\end{array}$ & $\begin{array}{l}\text { e. Sant.- } \\
\text { 1. Coniac. } \\
\text { e. Sant.- } \\
\text { 1. Coniac. }\end{array}$ & $\begin{array}{l}86.1 \\
86.5\end{array}$ \\
\hline \multicolumn{6}{|l|}{ Leg 39, Hole 357} \\
\hline $36-4,36-44$ & Missing ${ }^{b}$ & $?$ & $\begin{array}{l}\text { Green-gray marly } \\
\text { chalks and } \\
\text { limestones }\end{array}$ & Campanian & 70.6 \\
\hline $44-2,38-39$ & 1 & Poor & $\begin{array}{l}\text { Green-gray marly } \\
\text { chalks and } \\
\text { limestone }\end{array}$ & I. Santonian & 82.9 \\
\hline $44-2,103-104$ & 1 & Poor & $\begin{array}{l}\text { Green-gray marly } \\
\text { chalks and } \\
\text { limestone }\end{array}$ & 1. Santonian & 82.9 \\
\hline $44-2,110-111$ & 1 & Poor & $\begin{array}{l}\text { Green-gray marly } \\
\text { chalks and } \\
\text { limestone }\end{array}$ & 1. Santonian & 82.9 \\
\hline
\end{tabular}


Table 1. (Continued.)

\begin{tabular}{|c|c|c|c|c|c|}
\hline $\begin{array}{l}\text { Core-Section } \\
\text { (interval in cm) }\end{array}$ & $\begin{array}{c}\text { No. of } \\
\text { occurrences }\end{array}$ & $\begin{array}{l}\text { Preservation } \\
\text { (visual exam.) }\end{array}$ & Sediment type & Stage & $\begin{array}{c}\text { Age } \\
\text { (m.y. ago) }\end{array}$ \\
\hline \multicolumn{6}{|c|}{ Leg 39, Hole 357 Cont. } \\
\hline $44-3,24-25^{\mathrm{a}}$ & 2 & Moderate & $\begin{array}{l}\text { Green-gray marly } \\
\text { chalks and } \\
\text { limestone }\end{array}$ & 1. Santonian & 82.9 \\
\hline $45-2,97-99$ & 1 & Poor & $\begin{array}{l}\text { Green-gray marly } \\
\text { chalks and } \\
\text { limestones }\end{array}$ & 1. Santonian & 83.3 \\
\hline $45-2,111-112$ & 2 & Poor & $\begin{array}{l}\text { Green-gray marly } \\
\text { chalks and } \\
\text { limestones }\end{array}$ & 1. Santonian & 83.3 \\
\hline $46-1,125-126$ & 1 & Poor & $\begin{array}{l}\text { Green-gray marly } \\
\text { chalks and } \\
\text { limestones }\end{array}$ & 1. Santonian & 83.3 \\
\hline $46-1,133-134$ & 1 & Poor & $\begin{array}{l}\text { Green-gray marly } \\
\text { chalks and } \\
\text { limestones }\end{array}$ & I. Santonian & 83.3 \\
\hline $46-2,92-96$ & Several & Poor & $\begin{array}{c}\text { Green-gray marly } \\
\text { chalks and } \\
\text { limestones }\end{array}$ & 1. Santonian & 83.4 \\
\hline $46-2,139-140^{a}$ & 1 & Moderate & $\begin{array}{l}\text { Green-gray marly } \\
\text { chalks and } \\
\text { limestones }\end{array}$ & 1. Santonian & 83.4 \\
\hline $46-3,3-4$ & 1 & Poor & $\begin{array}{l}\text { Green-gray marly } \\
\text { chalks and } \\
\text { limestones }\end{array}$ & I. Santonian & 83.4 \\
\hline $47-1,143-144$ & 1 & Poor & $\begin{array}{l}\text { Green-gray marly } \\
\text { chalks and } \\
\text { limestones }\end{array}$ & I. Santonian & 83.7 \\
\hline $47-2,2-3$ & 1 & Poor & $\begin{array}{l}\text { Green-gray marly } \\
\text { chalks and } \\
\text { limestones }\end{array}$ & I. Santonian & 83.7 \\
\hline $47-2,11-12$ & 1 & Poor & $\begin{array}{l}\text { Green-gray marly } \\
\text { chalks and } \\
\text { limestones }\end{array}$ & 1. Santonian & 83.7 \\
\hline $47-2,137-138$ & 1 & Poor & $\begin{array}{l}\text { Green-gray marly } \\
\text { chalks and } \\
\text { limestones }\end{array}$ & I. Santonian & 83.7 \\
\hline $47-3,13-15$ & 1 & Poor & $\begin{array}{l}\text { Green-gray marly } \\
\text { chalks and } \\
\text { limestones }\end{array}$ & 1. Santonian & 83.7 \\
\hline $47-3,48-49$ & 1 & Poor & $\begin{array}{l}\text { Green-gray marly } \\
\text { chalks and } \\
\text { limestones }\end{array}$ & I. Santonian & 83.7 \\
\hline $47-3,51-52$ & 1 & Poor & $\begin{array}{l}\text { Green-gray marly } \\
\text { chalks and } \\
\text { limestones }\end{array}$ & I. Santonian & 83.7 \\
\hline $47-4,41-42$ & 1 & Poor & $\begin{array}{c}\text { Green-gray marly } \\
\text { chalks and } \\
\text { limestones }\end{array}$ & 1. Santonian & 83.8 \\
\hline $47-4,85-86$ & 1 & Poor & $\begin{array}{l}\text { Green-gray marly } \\
\text { chalks and } \\
\text { limestones }\end{array}$ & 1. Santonian & 83.8 \\
\hline $47-4,106-107$ & 1 & Poor & $\begin{array}{l}\text { Green-gray marly } \\
\text { chalks and } \\
\text { limestones }\end{array}$ & 1. Santonian & 83.8 \\
\hline $47-4,135-136$ & 1 & Poor & $\begin{array}{l}\text { Green-gray marly } \\
\text { chalks and } \\
\text { limestones }\end{array}$ & 1. Santonian & 83.8 \\
\hline $47-4,140-142$ & 1 & Poor & $\begin{array}{l}\text { Green-gray marly } \\
\text { chalks and } \\
\text { limestones }\end{array}$ & 1. Santonian & 83.8 \\
\hline $47-4,146-147$ & 1 & Poor & $\begin{array}{l}\text { Green-gray marly } \\
\text { chalks and } \\
\text { limestones }\end{array}$ & 1. Santonian & 83.9 \\
\hline $47-5,60-61$ & Several & Poor & $\begin{array}{l}\text { Green-gray marly } \\
\text { chalks and } \\
\text { limestones }\end{array}$ & I. Santonian & 83.9 \\
\hline $47-5,66-67$ & 1 & Poor & $\begin{array}{l}\text { Green-gray marly } \\
\text { chalks and } \\
\text { limestones }\end{array}$ & 1. Santonian & 83.9 \\
\hline $47-5,73-74$ & 1 & Poor & $\begin{array}{l}\text { Green-gray marly } \\
\text { chalks and } \\
\text { limestones }\end{array}$ & 1. Santonian & 83.9 \\
\hline $47-6,10-11$ & 1 & Poor & $\begin{array}{l}\text { Green-gray marly } \\
\text { chalks and } \\
\text { limestones }\end{array}$ & I. Santonian & 83.9 \\
\hline $47-6,13-14$ & 1 & Poor & $\begin{array}{l}\text { Green-gray marly } \\
\text { chalks and } \\
\text { limestones }\end{array}$ & I. Santonian & 83.9 \\
\hline $47-6,60$ & 1 & Poor & $\begin{array}{l}\text { Green-gray marly } \\
\text { chalks and } \\
\text { limestones }\end{array}$ & I. Santonian & 83.9 \\
\hline $47-6,61$ & 1 & Poor & $\begin{array}{l}\text { Green-gray marly } \\
\text { chalks and } \\
\text { limestones }\end{array}$ & I. Santonian & 83.9 \\
\hline $47-6,62$ & 1 & Poor & $\begin{array}{l}\text { Green-gray marly } \\
\text { chalks and } \\
\text { limestones }\end{array}$ & 1. Santonian & 83.9 \\
\hline $47-6,63-64^{a}$ & 1 & Moderate & $\begin{array}{l}\text { Green-gray marly } \\
\text { chalks and } \\
\text { limestones }\end{array}$ & 1. Santonian & 83.9 \\
\hline
\end{tabular}


Table I. (Continued.)

\begin{tabular}{|c|c|c|c|c|c|}
\hline $\begin{array}{l}\text { Core-Section } \\
\text { (interval in cm) }\end{array}$ & $\begin{array}{c}\text { No. of } \\
\text { occurrences }\end{array}$ & $\begin{array}{l}\text { Preservation } \\
\text { (visual exam.) }\end{array}$ & Sediment type & Stage & $\begin{array}{c}\text { Age } \\
\text { (m.y. ago) }\end{array}$ \\
\hline \multicolumn{6}{|c|}{ Leg 39, Hole 357 Cont. } \\
\hline $48-1,78-79$ & 1 & Poor & $\begin{array}{l}\text { Green-gray marly } \\
\text { chalks and } \\
\text { limestones }\end{array}$ & e. Santonian & 84.1 \\
\hline $48-1,79-80$ & 1 & Poor & $\begin{array}{l}\text { Green-gray marly } \\
\text { chalks and } \\
\text { limestones }\end{array}$ & e. Santonian & 84.1 \\
\hline $48-1,83-87$ & 1 & Poor & $\begin{array}{l}\text { Green-gray marly } \\
\text { chalks and } \\
\text { limestones }\end{array}$ & e. Santonian & 84.1 \\
\hline $48-2,136-137$ & Several & Poor & $\begin{array}{l}\text { Green-gray marly } \\
\text { chalks and } \\
\text { limestones }\end{array}$ & e. Santonian & 84.2 \\
\hline $48-3,35-36$ & Several & Poor & $\begin{array}{l}\text { Green-gray marly } \\
\text { chalks and } \\
\text { limestones }\end{array}$ & e. Santonian & 84.2 \\
\hline $48-3,114-117$ & 1 & Poor & $\begin{array}{l}\text { Green-gray marly } \\
\text { chalks and } \\
\text { limestones }\end{array}$ & e. Santonian & 84.3 \\
\hline $48-4,97-98$ & 1 & Poor & $\begin{array}{l}\text { Green-gray marly } \\
\text { chalks and } \\
\text { limestones }\end{array}$ & e. Santonian & 84.4 \\
\hline $48-4,104-105$ & 1 & Poor & $\begin{array}{l}\text { Green-gray marly } \\
\text { chalks and } \\
\text { limestones }\end{array}$ & e. Santonian & 84.4 \\
\hline $48-6,122-123$ & 1 & Poor & $\begin{array}{l}\text { Green-gray marly } \\
\text { chalks and } \\
\text { limestones }\end{array}$ & e. Santonian & 84.6 \\
\hline $49-3,25-26$ & 1 & Poor & $\begin{array}{l}\text { Green-gray marly } \\
\text { chalks and } \\
\text { limestones }\end{array}$ & e. Santonian & 84.9 \\
\hline $49-6,57-85^{a}$ & 1 & Poor & $\begin{array}{c}\text { Green-gray marly } \\
\text { chalks and } \\
\text { limestones }\end{array}$ & e. Santonian & 85.3 \\
\hline $50-1,0-2^{\mathrm{a}}$ & Several & Poor & $\begin{array}{l}\text { Green-gray marly } \\
\text { chalks and } \\
\text { limestones }\end{array}$ & e. Santonian & 85.3 \\
\hline $50-1,25-26$ & 1 & Poor & $\begin{array}{l}\text { Green-gray marly } \\
\text { chalks and } \\
\text { limestones }\end{array}$ & e. Santonian & 85.3 \\
\hline $50-1,102-103$ & Several & Poor & $\begin{array}{l}\text { Green-gray marly } \\
\text { chalks and } \\
\text { limestones }\end{array}$ & e. Santonian & 85.4 \\
\hline $50-2,65-66$ & 1 & Poor & $\begin{array}{l}\text { Green-gray marly } \\
\text { chalks and } \\
\text { limestones }\end{array}$ & e. Santonian & 85.4 \\
\hline $50-2,108-109$ & 1 & Poor & $\begin{array}{l}\text { Green-gray marly } \\
\text { chalks and } \\
\text { limestones }\end{array}$ & e. Santonian & 85.5 \\
\hline $50-2,121-122$ & 1 & Silicified & $\begin{array}{l}\text { Green-gray marly } \\
\text { chalks and } \\
\text { limestones }\end{array}$ & e. Santonian & 85.5 \\
\hline $50-4,37-38$ & 1 & Poor & $\begin{array}{l}\text { Olive marly } \\
\text { limestones }\end{array}$ & e. Santonian & 85.6 \\
\hline $50-4,116-117$ & 1 & Poor & $\begin{array}{l}\text { Olive marly } \\
\text { limestones }\end{array}$ & e. Santonian & 85.7 \\
\hline $50-4,137-138$ & 1 & Poor & $\begin{array}{l}\text { Olive marly } \\
\text { limestones }\end{array}$ & e. Santonian & 85.7 \\
\hline $50-4,139-140$ & 1 & Poor & $\begin{array}{l}\text { Olive marly } \\
\text { limestones }\end{array}$ & e. Santonian & 85.7 \\
\hline $50-6,108-109$ & Several & Silicified & $\begin{array}{l}\text { Olive marly } \\
\text { limestones }\end{array}$ & e. Santonian & 85.9 \\
\hline $50-6,113-114$ & Several & Silicified & $\begin{array}{l}\text { Olive marly } \\
\text { limestones }\end{array}$ & c. Santonian & 85.9 \\
\hline \multicolumn{6}{|l|}{ Leg 40, Hole 361} \\
\hline $15, \mathrm{CC},-$ & 1 & Moderate & Euxinic & e. Maes.-1. Camp. & 74.0 \\
\hline \multicolumn{6}{|l|}{ Leg 40, Hole 363} \\
\hline $21-3,133-134^{a}$ & 1 & Moderate & $\begin{array}{l}\text { Foram-nanno } \\
\text { chalk }\end{array}$ & e. Maestrichtian & 69.3 \\
\hline $21-6,48-52^{a}$ & 1 & Moderate & $\begin{array}{l}\text { Foram-nanno } \\
\text { chalk }\end{array}$ & e. Maestrichtian & 70.2 \\
\hline $21-6,52^{a}$ & 1 & Moderate & $\begin{array}{l}\text { Foram-nanno } \\
\text { chalk }\end{array}$ & e. Maestrichtian & 70.2 \\
\hline $21-6,67-68^{a}$ & 1 & Moderate & $\begin{array}{l}\text { Foram-nanno } \\
\text { chalk }\end{array}$ & e. Maestrichtian & 70.2 \\
\hline \multicolumn{6}{|l|}{ Leg 40, Hole 364} \\
\hline $38-3,24-25$ & 1 & Poor & $\begin{array}{l}\text { Olive marly } \\
\text { limestones }\end{array}$ & u. Aptian & 110.2 \\
\hline 38-3, 61-62 & 1 & Poor & $\begin{array}{l}\text { Olive marly } \\
\text { limestones }\end{array}$ & u. Aptian & 110.3 \\
\hline $39-5,20-22^{\mathrm{a}}$ & 1 & Good & $\begin{array}{l}\text { Olive marly } \\
\text { limestones }\end{array}$ & u. Aptian & 111.8 \\
\hline \multicolumn{6}{|l|}{ Leg 75 , Hole $530 \mathrm{~A}$} \\
\hline $55-1,11-12$ & $\begin{array}{l}1 \\
3\end{array}$ & $\begin{array}{l}\text { Silicified } \\
\text { Silicified }\end{array}$ & Green mudstone & e. Maes.-1. Camp. & 70.0 \\
\hline $55-1,34-36$ & & Silicified & Green mudstone & e. Maes.-1. Camp. & 70.0 \\
\hline
\end{tabular}


Table 1. (Continued.)

\begin{tabular}{|c|c|c|c|c|c|}
\hline $\begin{array}{l}\text { Core-Section } \\
\text { (interval in cm) }\end{array}$ & $\begin{array}{c}\text { No. of } \\
\text { occurrences }\end{array}$ & $\begin{array}{l}\text { Preservation } \\
\text { (visual exam.) }\end{array}$ & Sediment type & Stage & $\begin{array}{c}\text { Age } \\
\text { (m.y. ago) }\end{array}$ \\
\hline \multicolumn{6}{|c|}{ Leg 75, Hole 530A Cont. } \\
\hline $55-1,39-41$ & 2 & Silicified & Green mudstone & e. Maes.-1. Camp. & 70.0 \\
\hline $55-1,67-68$ & 1 layer & Silicified & Green mudstone & e. Maes. -1 . Camp. & 70.0 \\
\hline $55-1,141-143$ & 3 & Silicified & Green mudstone & c. Maes,-1. Camp. & 70.0 \\
\hline $55-2,68-69$ & 1 & Silicified & Green mudstone & c. Maes. -1. Camp. & 70.0 \\
\hline $55-2,105-106$ & i & Silicified & Green mudstone & e. Maes.-1. Camp. & 70.2 \\
\hline $55-3,112-113$ & 1 & Silicified & Green mudstone & e. Maes.-I. Camp. & 70.2 \\
\hline $56-2,44-45$ & 2 & Silicified & Green mudstone & c. Maes.-I. Camp. & 70.6 \\
\hline $56-2,45-46$ & $i$ & Silicified & Green mudstone & c. Maes.-1. Camp. & 70.6 \\
\hline $56-2,48-49$ & $i$ & Silicified & Green mudstone & c. Maes.-1. Camp. & 70.6 \\
\hline $56-2,56-58$ & 1 & Silicified & Green mudstone & e. Maes.-1. Camp. & 70.6 \\
\hline $56-2,62-63$ & 1 & Silicified & Green mudstone & e. Maes.-I. Camp. & 70.6 \\
\hline $56-2,69-70$ & 1-layer & Silicified & Green mudstone & e. Maes.-I. Camp. & 70.6 \\
\hline $56-2,87-88$ & 1 & Silicified & Green mudstone & e. Maes.-1. Camp. & 70,6 \\
\hline $56, \mathrm{CC}$ & $i$ & Silicified & Green mudstone & e. Maes.-1. Camp. & 70.6 \\
\hline $60-1,121-124^{\mathrm{a}}$ & Several & Good & Green mudstone & e. Maes.-I. Camp. & 70.8 \\
\hline $61-2,53-54^{\mathrm{a}}$ & Several & Very good & $\begin{array}{l}\text { Green mudstone/ } \\
\text { marlstone }\end{array}$ & e. Maes.-1. Camp. & 70.9 \\
\hline $61-2,56-57^{a}$ & Several & Very good & $\begin{array}{l}\text { Green mudstone/ } \\
\text { marlstone }\end{array}$ & e. Maes.-1. Camp. & 70.9 \\
\hline $61-2,65-66^{a}$ & 1 layer & Moderate & $\begin{array}{l}\text { Green mudstone/ } \\
\text { marlstone }\end{array}$ & e. Maes.-1. Camp. & 70.9 \\
\hline $61-2,78-81^{a}$ & 2 layers & Very good & $\begin{array}{l}\text { Green mudstone/ } \\
\text { marlstone }\end{array}$ & e. Maes.-1. Camp. & 70.9 \\
\hline $61-3,59-61$ & 1 & Poor & $\begin{array}{l}\text { Green mudstone/ } \\
\text { maristone }\end{array}$ & e. Maes.-1. Camp. & 71.5 \\
\hline $63-1,37-40^{\mathrm{a}}$ & 2 & Good & $\begin{array}{l}\text { Green mudstone/ } \\
\text { marlstone }\end{array}$ & e. Maes.-1. Camp. & 72.5 \\
\hline $63-2,71-72^{\mathrm{a}}$ & 1 & Poor & $\begin{array}{l}\text { Green mudstone/ } \\
\text { marlstone }\end{array}$ & e. Maes.-1. Camp. & 72.5 \\
\hline $63-2,79-80$ & 1 layer & Poor & $\begin{array}{l}\text { Green mudstone/ } \\
\text { marlstone }\end{array}$ & e. Maes.-1. Camp. & 72.5 \\
\hline $63-3,6-8$ & & Poor & $\begin{array}{l}\text { Green mudstone/ } \\
\text { marlstone }\end{array}$ & e. Maes.-1. Camp. & 72.7 \\
\hline $63-3,8-9$ & & Poor & $\begin{array}{l}\text { Green mudstone/ } \\
\text { marlstone }\end{array}$ & e. Maes.-1. Camp. & 72.7 \\
\hline $63-3,52-53$ & 1 layer & Poor & $\begin{array}{l}\text { Green mudstone/ } \\
\text { marlstone }\end{array}$ & e. Maes.-1. Camp. & 72.7 \\
\hline $63-3,104-105^{a}$ & Several & Poor & Sandy layer & e. Maes.-1. Camp. & 72.7 \\
\hline $64-1,113-114^{a}$ & 1 & Poor & $\begin{array}{l}\text { Olive mudstone/ } \\
\text { marlstone }\end{array}$ & Campanian & 73.1 \\
\hline $64-2,8-9^{a}$ & Several & Good & $\begin{array}{l}\text { Olive mudstone/ } \\
\text { marlstone }\end{array}$ & Campanian & 73.1 \\
\hline $64-2,10-11^{a}$ & Several & Good & $\begin{array}{l}\text { Olive mudstone/ } \\
\text { marlstone }\end{array}$ & Campanian & 73.1 \\
\hline $79-5,71-73^{\mathrm{a}}$ & 1 & Very good & Sandy layer & Campanian & 81.2 \\
\hline $79-5,141-142^{\mathrm{a}}$ & Several & Good & $\begin{array}{l}\text { Red and green } \\
\text { claystone }\end{array}$ & Campanian & 81.2 \\
\hline $80-1,1^{\mathrm{a}}$ & 1 layer & Moderate & $\begin{array}{l}\text { Red and green } \\
\text { claystone }\end{array}$ & Campanian & 81.9 \\
\hline $83-1,62-63^{a}$ & 1 & Good & Fine sand & $\begin{array}{l}\text { Santonian- } \\
\text { Coniac. }\end{array}$ & 83.6 \\
\hline $83-1,98-99$ & 1 & Poor & $\begin{array}{l}\text { Red and green } \\
\text { claystone }\end{array}$ & $\begin{array}{l}\text { Santonian- } \\
\text { Coniac. }\end{array}$ & 83.6 \\
\hline $83-1,123-124$ & 1 layer & Poor & $\begin{array}{l}\text { Red and green } \\
\text { claystone }\end{array}$ & $\begin{array}{l}\text { Santonian- } \\
\text { Coniac. }\end{array}$ & 83.6 \\
\hline $83-2,118-119^{a}$ & 1 & Moderate & $\begin{array}{l}\text { Red and green } \\
\text { claystone }\end{array}$ & $\begin{array}{l}\text { Santonian- } \\
\text { Coniac. }\end{array}$ & 83.7 \\
\hline $83-3,50-51$ & 1 & Poor & $\begin{array}{l}\text { Red and green } \\
\text { claystone }\end{array}$ & $\begin{array}{l}\text { Santonian- } \\
\text { Coniac. }\end{array}$ & 83.8 \\
\hline $83-3,112-114^{a}$ & 1 & Moderate & $\begin{array}{l}\text { Red and green } \\
\text { claystone }\end{array}$ & $\begin{array}{l}\text { Santonian- } \\
\text { Coniac. }\end{array}$ & 83.8 \\
\hline $83-4,5-6^{a}$ & I layer & Moderate & $\begin{array}{l}\text { Sedimentary } \\
\text { laminations }\end{array}$ & $\begin{array}{l}\text { Santonian- } \\
\text { Coniac. }\end{array}$ & 83.8 \\
\hline $83-4,33-34$ & 1 & Poor & Red claystones & $\begin{array}{l}\text { Santonian- } \\
\text { Coniac. }\end{array}$ & 83.9 \\
\hline $85-2,23-24^{a}$ & 1 & Poor & $\begin{array}{l}\text { Red-brown } \\
\text { claystones }\end{array}$ & $\begin{array}{l}\text { Santonian- } \\
\text { Coniac. }\end{array}$ & 84.9 \\
\hline $85-2,66-67 a$ & 2 & Moderate & $\begin{array}{l}\text { Red-brown } \\
\text { claystones }\end{array}$ & $\begin{array}{l}\text { Santonian- } \\
\text { Coniac. }\end{array}$ & 84.9 \\
\hline $85-3,4-5^{a}$ & 1 & Moderate to poor & $\begin{array}{l}\text { Red-brown } \\
\text { claystones }\end{array}$ & $\begin{array}{l}\text { Santonian- } \\
\text { Coniac. }\end{array}$ & 85.0 \\
\hline $85-3,30-31^{a}$ & Several & Moderate & $\begin{array}{l}\text { Red-brown } \\
\text { claystone }\end{array}$ & $\begin{array}{l}\text { Santonian- } \\
\text { Coniac. }\end{array}$ & 85.0 \\
\hline $88-2,94-95^{\mathrm{a}}$ & 3 & Cemented block & $\begin{array}{l}\text { Red and green } \\
\text { claystone }\end{array}$ & $\begin{array}{l}\text { Santonian- } \\
\text { Coniac. }\end{array}$ & 86.1 \\
\hline $89-1,88-89^{a}$ & 2 layers & Good & $\begin{array}{l}\text { Red and green } \\
\text { claystone }\end{array}$ & $\begin{array}{l}\text { Santonian- } \\
\text { Coniac. }\end{array}$ & 86.3 \\
\hline
\end{tabular}

a A sample.

See site summary, Leg 39. 
Table 2. Age-depth relationships used to calculate the age in millions of years for $I n$ oceramus, using the time scale of Obradovich and Cobban (1975) for the Late Cretaceous and van Hinte (1976a, b) for the Early Cretaceous and Jurassic, and a constant sedimentation rate within paleontologic zones described in the respective site reports.

\begin{tabular}{ccc}
\hline & $\begin{array}{c}\text { Sub-bottom } \\
\text { depth } \\
\text { Core }\end{array}$ & $\begin{array}{c}\text { Age } \\
\text { (m) }\end{array}$ \\
\hline
\end{tabular}

Leg 3, Hole 21

$\begin{array}{lcc}3 & 76.3-85.4 & 65.0-67.5 \\ 4 & 85.4-87.7 & 67.5-69.4 \\ 4-5 & 87.7-105.9 & 70.5-75.8 \\ 6-8 & 105.9-130.6 & 75.8-82.0\end{array}$

Leg 36, Hole 327A

(coring not continuous)

\begin{tabular}{lrl}
$10-12$ & $90.0-118.0$ & \multicolumn{1}{l}{$65.0-$} \\
13 & $137.0-142.0$ & -68.3 \\
13 & $142.0-146.5$ & $68.3-75.8$ \\
14 & $146.5-151.0$ & $85.0-86.0$ \\
14 & $151.0-156.0$ & $91.7-92.5$ \\
15 & $175.0-184.5$ & $94.0-100.0$
\end{tabular}

Leg 36, Hole 330

(coring not continuous)

$\begin{array}{llc}1 & 129.0-138.5 & 100.0- \\ 2 & 176.5-186.0 & -106.5 \\ 3 & 224.0-233.5 & 112.0- \\ 4 & 271.5-281.5 & -115.0 \\ 5-7 & 300.0-328.5 & 143.0- \\ 8 & 347.5-357.5 & -148.0\end{array}$

Leg 39, Hole 355

$17-18 \quad 404.5-423.5$

$19-21 \quad 423.5-449.0$

69.4-72.0

$72.0-82.0$

Leg 39, Hole 356

$\begin{array}{lll}34 & 513.0-522.5 & 75.8-82.0 \\ 35-37 & 541.5-608.0 & 82.0-84.0 \\ 38-40 & 646.5-700.0 & 84.0-86.5 \\ 40 & 700.0-703.0 & 87.8-88.6\end{array}$

Leg 39 Hole 357

$\begin{array}{lll}36-41 & 607.5-711.5 & 70.5-82.0 \\ 42-47 & 711.5-759.0 & 82.0-84.0 \\ 48-51 & 759.0-787.5 & 84.0-86.0\end{array}$

$\begin{array}{lll}48-51 & 759.0-787.5 & 84.0-86.0\end{array}$

Leg 40, Hole 361

$12-20 \quad 297.5-620.0 \quad 65.0-87.0$

Leg 40, Hole 363

$21 \quad 363.5-373.0$

$68.3-70.5$

Leg 40, Hole 364

$\begin{array}{llc}38 & 948.0-947.5 & 110.0- \\ 39 & 967.0-967.5 & -112.0\end{array}$

Leg 75 , Hole 530A

\begin{tabular}{lll}
$50-59$ & $590.5-685.5$ & $65.0-70.5$ \\
$59-80$ & $685.5-885.0$ & $70.5-82.0$ \\
$80-94$ & $885.0-1008.0$ & $82.0-87.0$ \\
\hline
\end{tabular}

volume) indicates that specimens were not displaced from shallower regions except possibly in three cases (Samples 530A-63-3, $104 \mathrm{~cm}$; 530A-79-5, $71 \mathrm{~cm}$; and 530A-83-1, $62 \mathrm{~cm}$ are associated with fine-grained sandy layers). All the DSDP cores were examined carefully for any characteristic which might indicate downslope transport in order to reconstruct the occurrence of Inoceramus and interpret the paleotemperature data accurately. It does not appear that Inoceramus is indicative of any specific paleodepth.

\section{STABLE ISOTOPE ANALYSES: OXYGEN AND CARBON}

\section{Analytical Techniques}

Oxygen and carbon isotopic compositions were measured by analyzing $\mathrm{CO}_{2}$ evolved by the reaction of samples and standards (B-1 and NBS-20) with $100 \% \mathrm{H}_{3} \mathrm{PO}_{4}$ at $40^{\circ} \mathrm{C}$. Analyses were performed on a Micromass 602 ratio mass spectrometer. The measurement technique has standard reproducibility of better than $\pm 0.1 \%$. All analyses are reported relative to PDB (Pee Dee Belemnite).

Paleotemperatures are calculated using the equation of Craig (1965). The oxygen isotopic composition of mean.ocean water is taken to be $-1.0 \%$ relative to SMOW (Standard Mean Ocean Water), assuming polar ice volume to be negligible during the Cretaceous (Shackleton and Kennett, 1975).

\section{Sample Preparation}

All samples were taken from the calcitic prismatic layer of Inoceramus fragments either by simply breaking off pieces, prying free individual crystals, or by drilling small holes in the shell with a highspeed burr. No detectable isotope effects are associated with the drilling procedure.

\section{DIAGENETIC TRENDS}

Isotopic results from 25 Inoceramus fragments are listed in Table 4 and plotted in Figure 3. Eleven samples with no evidence of recrystallization, dissolution, overgrowths, or silicification are indicated as "well preserved" in Figure 3. These samples exhibit a range in $\delta^{18} \mathrm{O}$ of +1.1 to $-2.6 \%$ and a range of +1.9 to -1.25 in $\delta^{13} \mathrm{C}$ and were considered suitable for paleotemperature analysis. Several of the moderately well-preserved samples also fall within these ranges. Poorly preserved samples tend to be lighter in both oxygen and carbon, reaching values of $-6.75 \%$ in $\delta^{18} \mathrm{O}$ and -3.2 in $\delta^{13} \mathrm{C}$.

Depletion in ${ }^{18} \mathrm{O}$ with increasing textural alteration is a common diagenetic feature in deep sea sediments. This is further illustrated by analyses of bulk carbonate from a black shale (Sample 530A-88-3, $122 \mathrm{~cm}$ ) of Coniacian age. The carbonate component of the black shale consisted largely of recrystallized foraminifers with calcitic overgrowths. The $\delta^{18} \mathrm{O}$ of the sample is $-7.57 \%$. The observed depletion in $\mathrm{O}^{18}$ of diagenetic carbonates may result from two effects: (1) increasing temperature with burial depth, and (2) pore water ${ }^{18} \mathrm{O}$ depletion resulting from formation of authigenic silicates.

The oxygen isotopic composition of the diagenetic carbonates $(-4.5$ to $-7.5 \%)$ yields a calculated temperature of $33-50^{\circ} \mathrm{C}$. This agrees reasonably well with the $30-45^{\circ} \mathrm{C}$ estimated downhole temperature of these sediments (Site 530 summary chapter, this volume).

Lawrence et al. (1975) found a correlation of $\delta^{18} \mathrm{O}$ gradients with changes in pore water cation composition and mineralogy at several DSDP sites. They demon- 



Figure 2. A. Occurrence of Inoceramus in Late Cretaceous sediments in South Atlantic DSDP cores, with age in million years. Each dot represents a single Inoceramus fragment. Site 364 has a Late Cretaceous section but is not plotted because there is no record of Inoceramus at this time. Site 361 has a record of the late Aptian to the Early Cretaceous and Site 356 has Turonian and late Albian sediments, but are not plotted because there is no record of Inoceramus at these times. Isolated calcite prisms are not included. The stratigraphy is given in Table 2. B. The occurrence of Inoceramus in Jurassic and middle Cretaceous sediments in South Atlantic DSDP cores. Each dot represents a single Inoceramus fragment. Isolated calcite prisms are not included. The stratigraphy is given in Table 2.

Table 3. Estimated paleodepths for South Atlantic DSDP sites with Inoceramus, based on the respective site reports. A range of paleodepth from oldest to youngest occurrence is given.

\begin{tabular}{llc}
\hline Hole & \multicolumn{1}{c}{ Stage } & $\begin{array}{c}\text { Water } \\
\text { depth } \\
\text { (m) }\end{array}$ \\
\hline 21 & Campanian & $\approx 500$ \\
$327 \mathrm{~A}$ & Albian-Maestrichtian & $100-400$ to 2000 \\
330 & Oxfordian-Albian & $<1500$ \\
355 & Campanian-E. Maestrichtian & 2500 to 3000 \\
356 & Albian & 1000 \\
357 & Santonian-Campanian & 1000 \\
361 & Campanian-E. Maestrichtian & 3000 to 4000 \\
363 & Maestrichtian & 1000 \\
364 & Aptian-Albian & $<2000$ \\
$530 \mathrm{~A}$ & Santonian-Maestrichtian & 3500 to 4500 \\
\hline
\end{tabular}

strated that alteration of volcanogenic sediments to more ${ }^{18} \mathrm{O}$ enriched authigenic phases is responsible for the pore water isotope shift. Volcanogenic turbidites of Campanian age are present in Unit 6 of Hole 530A and may have caused some pore water changes. Without pore water $\delta^{18} \mathrm{O}$ measurements, it is not possible to distinguish between the effects of temperature and pore water alteration on the $\delta^{18} \mathrm{O}$ of diagenetic carbonates.

\section{PALEOTEMPERATURE RESULTS}

Eleven well-preserved specimens were selected for paleotemperature analysis (Table 5). These specimens yield isotopic temperatures ranging from 11.3 to $23.9^{\circ} \mathrm{C}$. Isotopic variability within single specimens is generally slightly greater than analytical error. For example, nine analyses of a Campanian fragment $(79-5,140 \mathrm{~cm})$ give a $\delta^{18} \mathrm{O}$ of $-0.78 \pm 0.24 \%(1 \sigma)$ and $\mathrm{a} \delta^{13} \mathrm{C}$ of $1.21 \pm 0.08$ $\%$ o $(1 \sigma)$. The calculated paleotemperature for this specimen is $16 \pm 1^{\circ} \mathrm{C}$.

Three of the specimens were associated with sandy layers, raising the possibility of downslope transport. Shallow water indicators are notably absent from these sediments. Hence it is impossible to determine how far the fragments may have been transported. Two of these specimens $(79-5,72 \mathrm{~cm}$ and $83-1,62-63 \mathrm{~cm})$ yield isotopic temperatures which are slightly warmer than most in the core $\left(23.9\right.$ and $19.2^{\circ} \mathrm{C}$, respectively). However, Sample 530A-89-1, 88-89 cm of Coniacian age, which showed no evidence of transport, gave a similar temperature $\left(23.1^{\circ} \mathrm{C}\right)$. The third fragment $(63-3,104-105 \mathrm{~cm})$, associated with a sandy layer, yielded one of the coldest temperatures in the core $\left(11.5^{\circ} \mathrm{C}\right)$ and was probably not transported from very far upslope. The remainder of the specimens in Table 5 are considered to be in situ and 
Table 4. Oxygen and carbon isotopic results from Inoceramus, Hole 530A, Leg 75 , all samples.

\begin{tabular}{|c|c|c|}
\hline $\begin{array}{l}\text { Core-Section } \\
\text { (interval in } \mathrm{cm} \text { ) }\end{array}$ & $\delta^{18} \mathrm{O}_{\mathrm{PDB}}$ & $\delta^{13} \mathrm{C}_{\mathrm{PDB}}$ \\
\hline \multirow[t]{2}{*}{$60-1,121-124$} & -0.40 & 0.78 \\
\hline & -0.27 & 0.74 \\
\hline $61-2,53-54$ & 0.40 & 0.88 \\
\hline $61-2,56-57$ & -0.17 & 0.59 \\
\hline $61-2,65-66$ & -0.91 & 1.01 \\
\hline \multirow[t]{2}{*}{$61-2,78-81$} & -0.90 & 1.38 \\
\hline & -0.82 & 1.21 \\
\hline \multirow[t]{4}{*}{$63-1,37-40$} & -0.60 & 0.40 \\
\hline & -0.93 & 0.55 \\
\hline & 0.20 & 1.01 \\
\hline & 0.156 & 0.324 \\
\hline $63-2,71-72$ & - & - \\
\hline $63-3,104-105$ & 0.35 & 0.38 \\
\hline $64-1,113-114$ & -1.19 & -0.89 \\
\hline $64-2,9-10$ & -1.13 & -0.64 \\
\hline \multirow[t]{2}{*}{$64-2,10-11$} & -0.90 & -0.22 \\
\hline & -1.36 & -0.49 \\
\hline \multirow[t]{3}{*}{$79-5,72$} & -2.42 & 1.99 \\
\hline & -2.53 & 2.07 \\
\hline & -2.59 & 1.94 \\
\hline \multirow[t]{10}{*}{$79-5,140$} & -0.92 & \\
\hline & -0.58 & 1.16 \\
\hline & -1.01 & 1.30 \\
\hline & -0.83 & 1.35 \\
\hline & -1.01 & 1.19 \\
\hline & -0.80 & 1.19 \\
\hline & -0.62 & 1.21 \\
\hline & -0.53 & 1.15 \\
\hline & -0.47 & 1.07 \\
\hline & -1.14 & 1.25 \\
\hline $79-5,141-142$ & -2.03 & 0.94 \\
\hline $80-1,1$ & -2.82 & 1.10 \\
\hline \multirow[t]{3}{*}{$83-1,62-63$} & -1.53 & 0.84 \\
\hline & -1.66 & 0.79 \\
\hline & -1.53 & -0.68 \\
\hline \multirow[t]{2}{*}{$83-2,122-123$} & -5.02 & -0.18 \\
\hline & -4.61 & -0.41 \\
\hline \multirow[t]{3}{*}{$83-3,113$} & -0.48 & 1.23 \\
\hline & -1.97 & 0.57 \\
\hline & -0.62 & 1.19 \\
\hline \multirow{3}{*}{$83-4,5-6$} & -6.31 & -0.01 \\
\hline & -6.02 & -0.13 \\
\hline & -5.84 & -0.42 \\
\hline $85-2,23$ & -6.77 & -0.37 \\
\hline $85-2,66-67$ & -0.39 & 0.97 \\
\hline $85-3,4-5$ & -3.12 & -1.36 \\
\hline $85-3,30-31$ & -1.43 & -0.18 \\
\hline $88-2,94-95$ & -6.62 & -1.66 \\
\hline \multirow[t]{2}{*}{$89-1,88-89$} & -2.28 & -4.38 \\
\hline & -2.41 & -1.27 \\
\hline
\end{tabular}

suitable for interpretation of the paleotemperatures in the Angola Basin.

Estimated isotopic paleotemperatures from Table 5 are plotted as a function of time in Fig. 4. These results indicate that there was a general decrease in bottom water temperatures in the Angola Basin from the Coniacian to the late Campanian. Late Campanian samples show significant and apparently regular variability in a short section of core. These temperature variations appear to be real, though they are difficult to interpret in terms of physical processes.

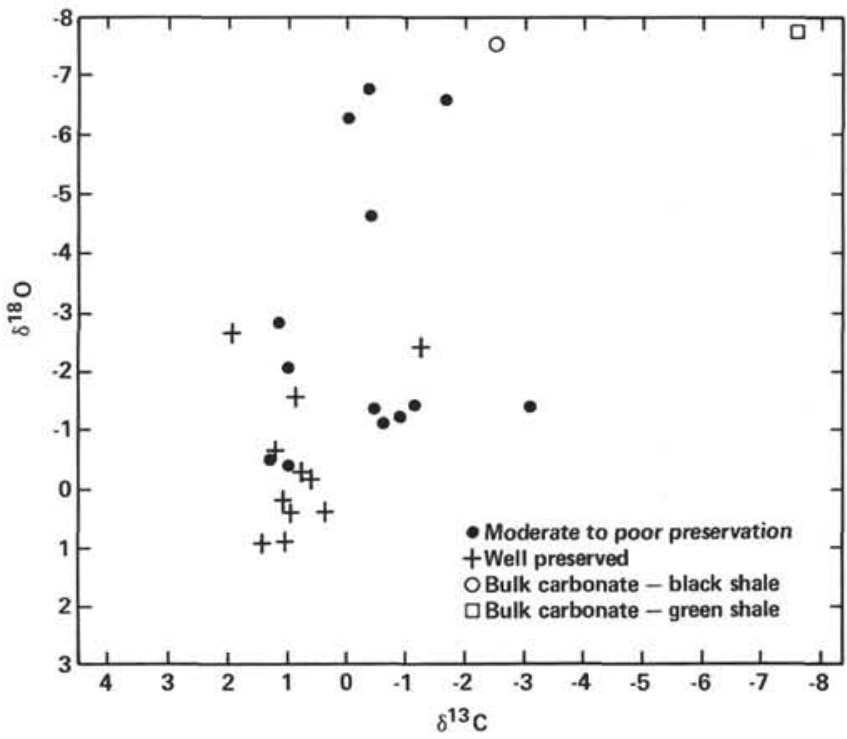

Figure 3. Carbon and oxygen isotopic results from all samples (indicated by footnote a in Table 1) at Hole 530A.

Table 5. Oxygen and carbon isotopic results from well-preserved Inoceramus, Hole 530A, Leg 75. Paleotemperatures are calculated using Craig's (1965) equation.

\begin{tabular}{crrr}
\hline $\begin{array}{c}\text { Core-Section } \\
\text { (interval in cm) }\end{array}$ & $\delta^{18}$ OPDB & ${ }^{13}{ }^{13}$ PDB & $\begin{array}{c}\mathrm{T} \\
\left({ }^{\circ} \mathrm{C}\right)\end{array}$ \\
\hline $60-1,121-124$ & -0.27 & 0.74 & 13.9 \\
$61-2,53-54$ & 0.40 & 0.88 & 11.3 \\
$56-57$ & -0.17 & 0.59 & 13.5 \\
$65-66$ & -0.91 & 1.01 & 16.5 \\
$78-81$ & -0.82 & 1.21 & 16.1 \\
$63-1,37-40$ & 0.20 & 1.01 & 12.1 \\
$63-3,104-105$ & 0.35 & 0.38 & 11.5 \\
$79-5,72$ & -2.59 & 1.94 & 23.9 \\
140 & -0.62 & 1.21 & 15.3 \\
$83-1,62-63$ & -1.53 & 0.68 & 19.2 \\
$89-1,88-89$ & -2.41 & -1.27 & 23.1 \\
\hline
\end{tabular}

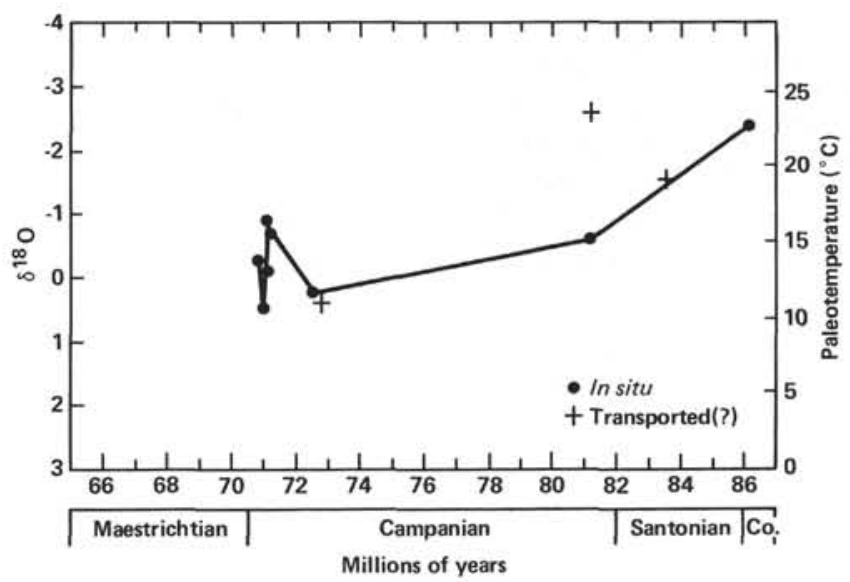

Figure 4. Oxygen isotopic paleotemperatures through time from all well-preserved specimens from Hole 530A. 


\section{DISCUSSION}

The Angola Basin is bounded by Africa, the Romanche Fracture Zone, the Mid-Atlantic Ridge, and the Walvis Ridge. The evolution of these topographic barriers strongly influenced the paleoceanography of the early South Atlantic. At present the topographic barriers have a large influence on the bottom water circulation. The Walvis Ridge is a barrier to bottom water circulation and only rather deep passages in the Rio Grande Rise permit overflow of Antarctic Bottom Water (AABW) into the Brazil Basin. The northward flow of the AABW is also significantly influenced by the mid-ocean ridges in both the Atlantic and Indian oceans (e.g., Neumann and Pierson, 1966).

During much of the Cretaceous, the Angola Basin was considerably more restricted than at present. For instance, sections of the Walvis Ridge were above sea level in the mid-Cretaceous (Sibuet et al., this volume). At this time the Walvis Ridge was probably a barrier to all but the surface circulation. Because of the proximity of Africa and South America at the Romanche Fracture Zone (e.g., Sclater et al., 1977), the connection to the North Atlantic was also considerably more restricted than at present. Thus we expect that the paleocirculation in the middle and Late Cretaceous was controlled more strongly by topography than at present. A second factor is that the African margin of the Angola Basin was characterized by extensive epicontinental seas during much of the Cretaceous, in contrast to the present day (e.g., Barron et al., 1981). Finally, from 100 to 70 m.y. ago most of the Angola Basin was confined to the subtropical latitudes (e.g., Barron and Harrison, 1980) and thus probably experienced relatively high evaporation rates. These three factors are likely to be the major controls of the Cretaceous paleoceanographic setting.

Based on the thermal subsidence history of oceanic crust and a basement age of about 102 m.y. at Site 530, the Coniacian sediments, containing the oldest recovered Inoceramus, probably were deposited at a depth greater than $3500 \mathrm{~m}$. The majority of the Inoceramus specimens from the Santonian and Campanian appar-

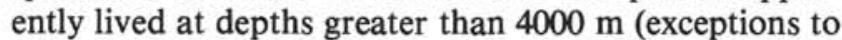
this were stated earlier). Thus, the paleotemperatures reflect paleoceanographic conditions in a deep restricted basin, bordered by epicontinental seas.

The measured isotopic temperatures are considerably warmer than those in present-day deep basins. The Coniacian value is extremely warm, implying a considerably reduced vertical temperature gradient in the basin. The basin is somewhat less restricted at the end of the Cretaceous, but the tectonic setting during the Coniacian and the Santonian-Campanian are similar. Since the paleolatitude of the basin had not changed, the difference in bottom temperature between the Coniacian and the Santonian-Campanian must reflect changes in the relative amount of colder and warmer bottom water sources during this time period.

The interpretation of the isotopic temperatures is further supported by Campanian isotopic measurements at Site 355 (Saltzman and Barron, 1982). Despite the fact that Site 355 has a shallower Campanian paleodepth than Site 530 , the Brazil Basin is about $5^{\circ} \mathrm{C}$ cooler than the Angola Basin. First, this clearly demonstrates that the Mid-Ocean Ridge is acting as an oceanographic barrier and second, the source, or mixing between sources of bottom water, must be different for the two basins. The bottom water in the Angola Basin must represent a greater proportion of water from a low latitude source. We speculate that warm, dense (saline) water was formed from high evaporation in the shallow epicontinental seas of Africa, and we note that the South American margin adjacent to the Brazil Basin is not characterized by extensive marginal seas. This supports the hypothesized processes of bottom water formation in subtropical latitudes as discussed by Brass et al. (1982).

These conclusions are important because they imply a source of warm saline bottom water for the Angola $\mathrm{Ba}$ sin and also indicate that mixing of cool and warm sources has probably increased toward the end of the Cretaceous. It is also important to note that if the water is saline, the isotopic temperature may be somewhat cooler than the actual temperature.

These results also have important implications for the sedimentation history in the South Atlantic. The isotopic paleotemperature for the Coniacian is the first recorded value that is stratigraphically associated with the deposition of black shales and implies relatively high bottom water temperatures. Warm temperatures, high salinity, and hence low oxygen solubility (Weiss, 1970) are likely to promote anoxia even without considering changes in productivity or mixing rates. Warmer temperatures in the Angola Basin, as compared to the Brazil Basin, are indicative of different bottom water sources and probably different oxygen contents.

The evolution of oceanic circulation in the South Atlantic was controlled by the tectonic setting, paleolatitude, and distribution of epicontinental seas. It is not surprising that anoxic sediments were not deposited synchronously in all the South Atlantic basins (see van Andel et al., 1977). Our data contribute to the picture of a dynamic, variable deep-water circulation in the Cretaceous South Atlantic.

\section{ACKNOWLEDGMENTS}

We wish to thank W. W. Hay, G. Brass, and C. Emiliani for their reviews of this manuscript. This research was supported by National Science Foundation grant OCE81-11909 to the University of Miami, Contribution No. 29 of the Harold C. Urey Laboratory for Isotopic Paleotemperature Research. The National Center for Atmospheric Research is sponsored by the National Science Foundation.

\section{REFERENCES}

Barron, E. J., and Harrison, C. G. A., 1980. An analysis of past plate motions: The South Atlantic and Indian oceans. In Davies, P. A., and Runcorn, S. K. (Eds.), Mechanisms of Continental Drift and Plate Tectonics: London (Academic Press), pp. 89-109.

Barron, E. J., Harrison, C. G. A., Sloan, J. L., II, and Hay, W. W., 1981. Paleogeography, 180 million years ago to the present. Eclog. Geol. Helvetae, 74:443-470.

Brass, G. W., Hay, W. W., Holser, W. T., Peterson, W. H., Saltzman, E., Sloan, J. L., II, and Southam, J. R., 1982. Ocean circulation, plate tectonics and climate. Pre-Pleistocene Climates: Washington (National Academy Press), pp. 83-89.

Craig, H., 1965. The measurement of oxygen isotope paleotemperatures. Stable Isotopes in Oceanographic Studies and Paleotemper- 
atures: Pisa (Consiglio Nazionale delle Ricerche, Laboratorio di Geologia Nucleare), pp. 1-24.

Kauffman, E. G., 1967. Coloradoan macroinvertebrate assemblages, central western interior, United States. In Kauffman, E. G., and Kent, H. C. (Eds.), Paleoenvironment of the Cretaceous SeawayA Symposium: Golden, Colorado (Colorado School of Mines), pp. 67-143.

1976. Deep-sea Cretaceous macrofossils: Hole 317A, Manihiki Plateau. In Jackson, E. D., Schlanger, S. O., et al., Init. Repts. DSDP, 33: Washington (U.S. Govt. Printing Office), 503-535.

Lawrence, J. R., Gieskes, J. M., and Broecker, W. S., 1975. Oxygen isotope and cation composition of DSDP pore waters and the alteration of Layer II basalts. Earth Planet. Sci. Letters, 27:1-10.

Lowenstam, H., and Epstein, S., 1954. Paleotemperatures of the postAptian Cretaceous as determined by oxygen isotope method $J$. Geol., 62:207-248.

Maxwell, A. E., Von Herzen, R. P., and Shipboard Scientific Party 1970. Site 21. In Maxwell, A. E., Von Herzen, R. P., et al., Init. Repts. DSDP, 3: Washington (U.S. Govt. Printing Office), $367-411$.

Neumann, G., and Pierson, W. J., 1966. Principles of Physical Oceanography: Englewood Cliffs, New Jersey (Prentice-Hall, Inc.).

Obradovich, J. D., and Cobban, W. A., 1975. A time scale for the the Late Cretaceous of the western interior of North America. Geol. Assoc. Can. Spec. Pap., 13:31-54.

Saito, T., and van Donk, J., 1974. Oxygen and carbon isotope measurements of Late Cretaceous and early Tertiary Foraminifera. Micropaleontology, 20:152-177.
Saltzman, E., Barron, E. J., and Price, D. A., 1982. South Atlantic Cretaceous paleotemperatures from DSDP cores. Palaeogeogr., Palaeoclim., Palaeoecol., 40:167-181.

Savin, A., 1977. The history of the earth's surface temperature during the past 100 million years. Ann. Rev. Earth Planet. Sci., 5: 319-355.

Sclater, J., Hellinger, S., and Tapscot, C., 1977. The paleobathymetry of the Atlantic Ocean from the Jurassic to the present. J. Geol., $85: 509-552$.

Shackleton, N. J., and Kennett, J. P., 1975. Paleotemperature history of the Cenozoic and the initiation of Antarctic glaciation: Oxygen and carbon isotope analyses in DSDP Sites 277,279 , and 281 . In Kennett, J. P., Houtz, R. E,. et al., Init. Repts. DSDP, 29: Washington (U.S. Govt. Printing Office), 743-755.

Thiede, J., and Dinkelman, M. G., 1977. Occurrence of Inoceramus remains in late Mesozoic pelagic and hemipelagic sediments. In Supko, P. R., Perch-Nielsen, K., et al., Init. Repts. DSDP, 39: Washington (U.S. Govt. Printing Office), 899-910.

Weiss, R. F., 1970. The solubility of nitrogen, oxygen and argon in water and seawater. Deep-Sea Res., 17:721-735.

van Andel, Tj. H., Thiede, J., Sclater, J. G., and Hay, W. W., 1977. Depositional history of the South Atlantic Ocean during the last 125 million years. J. Geol., 85:651-698.

van Hinte, J. E., 1976a. A Cretaceous time scale. Amer. Assoc. Petrol. Geol. Bull., 60:498-516.

1976b. A Jurassic time scale. Amer. Assoc. Petrol. Geol. Bull., 60:489-497.

Date of Initial Receipt: September 24, 1982 